\title{
Tunable positive magnetoresistance effect of Co-doped amorphous carbon films
}

\author{
Y. C. Jiang, Z. P. Wu, W. Bao, S. J. Xu, and J. Gao ${ }^{\text {a) }}$ \\ Department of Physics, The University of Hong Kong, Hong Kong, China
}

(Presented 31 October 2011; received 20 September 2011; accepted 4 November 2011; published online 1 March 2012)

Co-doped amorphous carbon (a-C:Co) films were deposited on $n$-type $\mathrm{Si}$ substrates by pulsed-laser deposition method. A positive magnetoresistance (PMR) effect has been observed after Co doped into a-C films. Such a PMR is tuned by the bias voltage and reaches a peak at a particular voltage, as observed from the Current-voltage relations of the a-C:Co/Si junctions at various magnetic fields. MR-H characteristics were further studied at the temperatures of $65 \mathrm{~K}$, which showed that under the reverse electric field the a-C:Co/Si junctions had a colossal PMR (over 100\%). Raman spectra results demonstrate that Co doping favors the formation of graphitic $\mathrm{sp}^{2}$ sites. The mechanism of the PMR effect is attributed to the interactions between the applied magnetic field and Co ions, which leads to the transition from $\mathrm{sp}^{2}$ sites to $\mathrm{sp}^{3}$ sites and increase the resistance. (C) 2012 American Institute of Physics. [doi:10.1063/1.3675180]

\section{INTRODUCTION}

Amorphous carbon (a-C) films have attracted great attention for the last decades because of their rich optical, magnetic, and electrical properties as well as possible applications for nonvolatile resistive switching memory, solar cells, gas sensors, magnetoresistance effect, and more. ${ }^{1-8}$ The a-C film has a disordered structure in which small $\mathrm{sp}^{2}$ clusters are embedded in the $\mathrm{sp}^{3}$ matrix. ${ }^{9,10}$ The $\mathrm{sp}^{2} / \mathrm{sp}^{3}$ ratio and the $\mathrm{sp}^{2}$ clustering size, influenced by various deposition conditions and different doping elements, are the two key matters determining its physical properties. ${ }^{11}$ Photovoltaic characteristics of Pd-doped a-C films and iodine-doped a-C films have been investigated and they showed high efficiency as solar cells. ${ }^{2,3}$ Gao et al. ${ }^{4}$ reported that a-C/Si junctions were sensitive to ammonia at room temperature, which showed a potential application as gas sensors. A positive magnetoresistance (PMR) effect was observed in iron-doped a-C films at room temperature, whereas the magnetoresistance turned negative below $260 \mathrm{~K} \cdot{ }^{5,6}$ All of these special properties of the a-C films derive from the interaction between doping elements and the two species of hybridized carbon atoms: $\mathrm{sp}^{2}$ and $\mathrm{sp}^{3}$

The colossal magnetoresistance (CMR) has been researched for several decades with applications for various electronic devices. ${ }^{12,13}$ The CMR effect is generally considered to originate from the Jahn-Teller effect and the double exchange. However, this mechanism is unlikely to be used to explain the colossal PMR effect of the disordered carbon systems. Further research is needed to clarify the transport mechanism of a-C films. The PMR effects of a-C:Co films and $\mathrm{a}-\mathrm{C}: \mathrm{Co} / \mathrm{Si}$ junctions have been observed at room temperature. $^{7,8}$ The PMR of the a-C:Co/Si junction reached $16 \%$ at magnetic field $2.5 \mathrm{kOe}$ and positive bias voltage

\footnotetext{
a) Author to whom correspondence should be addressed. Electronic mail: jugao@hku.hk.
}

$6 \mathrm{~V} .{ }^{8}$ However, the research on the PMR effect in the negative bias voltage still remains lacking. In our research, the a-C:Co films were deposited on silicon substrates and the p-n junctions were formed. The current-voltage relations measured at a low temperature $(65 \mathrm{~K})$ indicated that, in a negative bias voltage, a-C:Co films had much higher MR values than those in a positive bias voltage, which implies that there are two different species of PMR mechanisms dependent on the electric field direction.

\section{EXPERIMENTAL}

Pure a-C films and a-C:Co films were deposited on $n$-type (111) silicon substrates by the pulsed-laser deposition (PLD) method with the KrF excimer laser. ${ }^{14,15}$ The Si substrates are lightly $\mathrm{P}$ doped with resistivity of $8-13$ $\Omega \cdot \mathrm{cm}$. Before deposition, the Si substrates were ultrasonically cleaned in ethanol, then acetone, etched in diluted HF solution (10\%) for $15 \mathrm{~min}$, and rinsed in de-ionized water. The deposition chamber was pumped to $6 \times 10^{-6} \mathrm{mbar}$ and the substrates were heated to $400{ }^{\circ} \mathrm{C}$. A graphite disk with a purity $>99.99 \%$ embedded by a strip of cobalt (99.9\%) was used as the target. The deposition was performed by using a laser with energy of $360 \mathrm{~mJ} /$ pulse and frequency of $5 \mathrm{~Hz}$.

Scanning electron microscope (SEM) observation showed that the a-C:Co film had a smooth surface, and the thickness was about $260 \mathrm{~nm}$. Elemental composition and Co concentration were analyzed by energy-dispersive $\mathrm{x}$-ray spectroscopy (EDX). Raman spectroscopy was used to identify the disordered structures and measure the $\mathrm{sp}^{2} / \mathrm{sp}^{3}$ ratio. Silver surface electrodes were evaporated on a-C films to ensure the low Ohmic contact. The resistance, currentvoltage $(I-V)$ relations and magnetoresistance of a-C:Co/Si junctions were measured by using a two-probe method with a Keithley 2400 Source Meter. The magnetic field was provided by a superconductor magnet. 


\section{RESULTS AND DISCUSSION}

The SEM image of the surface of a-C:Co/Si junction is shown in Fig. 1(a), which demonstrates a smooth surface of the formed a-C:Co film. As shown by the cross-sectional SEM image of the a-C:Co/Si junction in Fig. 1(b), its thickness is quite uniform and is around $260 \mathrm{~nm}$. The EDX spectrum in Fig. 1(c) confirms that Co has been successfully doped into the a-C film. The doping concentration of $\mathrm{Co}$ reaches $12 \%$ in our sample. The research of Zhang et al. ${ }^{8}$ shows that the Co spikes are penetrated into $\mathrm{Si}$ substrates and distributed discontinuously at the $\mathrm{C} / \mathrm{Si}$ interface instead of dispersing in carbon film. The structural change of the $\mathrm{C} / \mathrm{Si}$ interface has a great effect on electrical and magnetic properties of a-C:Co/Si junctions.

Raman spectroscopy is a common method to analyze the disordered microstructure and the $\mathrm{sp}^{2} / \mathrm{sp}^{3}$ ratios of a-C films. There are two Raman active modes in the disordered carbon systems such as $\mathrm{G}$ mode (centered at $\sim 1550 \mathrm{~cm}^{-1}$ ) and $\mathrm{D}$ mode (centered at $\sim 1350 \mathrm{~cm}^{-1}$ ). The $\mathrm{G}$ peak is associated with the optically allowed E2 $\mathrm{g}$ zone center mode of crystalline graphite, whereas the D peak is associated with disorder-allowed zone-edge modes of graphite. ${ }^{16}$ In fact, the ratio of $D$ peak and $G$ peak intensities is consistent with the ratio of $\mathrm{sp}^{2}$ and $\mathrm{sp}^{3}$ sites. Figure 2 shows the Raman spectra excited by $514 \mathrm{~nm}$ laser for the pure a-C film and the a-C:Co film at room temperature. The two peaks ( $G$ and $D)$ have been observed, which reveals that the samples are typical amorphous carbon systems. The $\mathrm{sp}^{2} / \mathrm{sp}^{3}$ ratio increases apparently with Co doping in the a-C film, which shows that Co doping favors the formation of $\mathrm{sp}^{2}$ clusters.

Figure 3(a) shows $I-V$ characteristics of a-C:Co/Si junction at various temperatures. The inset displays the schematic illustration of the electrical measurement. It is observed that only when the temperature is above $270 \mathrm{~K}$, will the $I-V$ curves have good rectifying behaviors, which

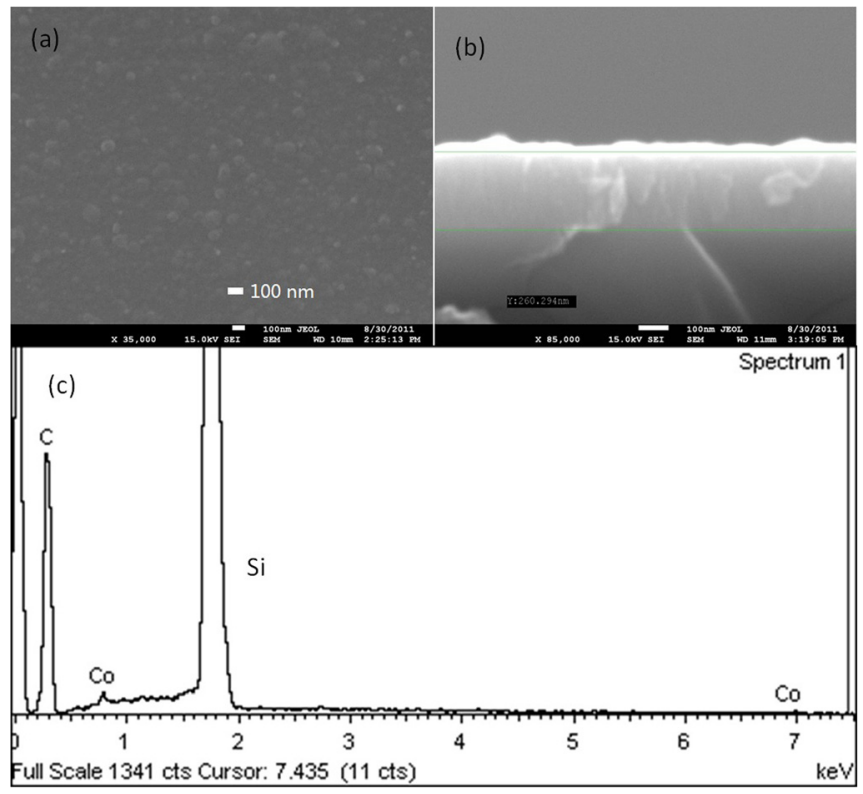

FIG. 1. (Color online) (a) The SEM image of the a-C:Co film deposited on $\mathrm{Si}$ substrate at $400{ }^{\circ} \mathrm{C}$. (b) The cross-sectional SEM image of the a-C:Co/Si junction. (c) EDX spectrum of the a-C:Co/Si junction.

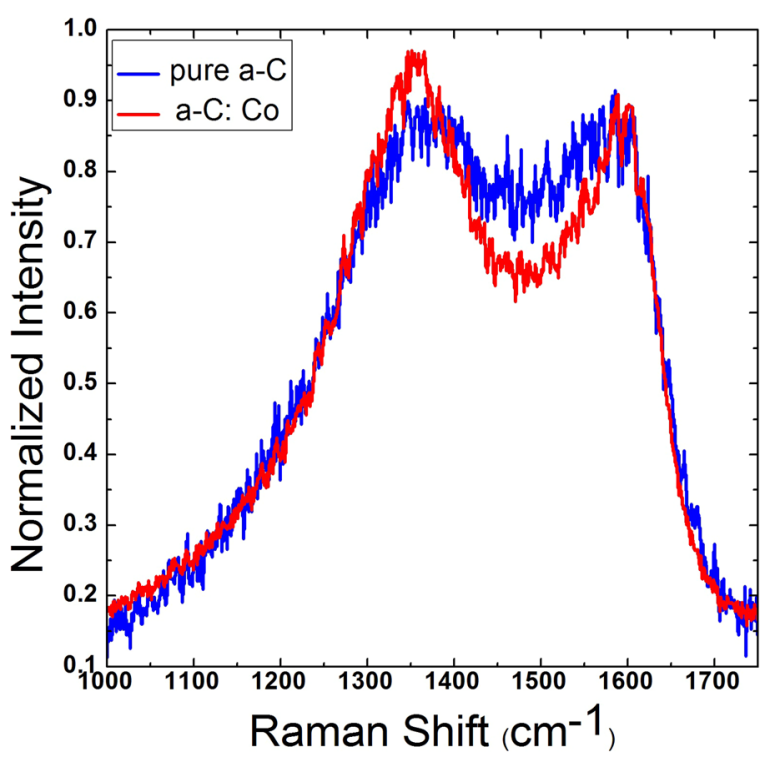

FIG. 2. (Color online) Raman spectra of the pure a-C film and the a-C:Co film deposited on Si substrates at room temperature.

differs from the result that pure a-C/Si junctions showed good rectifying properties in a large temperature range $(80-300 \mathrm{~K}){ }^{17}$ In addition, when the negative voltage overcomes a threshold $(5.5 \mathrm{~V})$, the reverse current increases sharply, but still greatly depends on temperature. The result illustrates that the breakdown voltage increases as the temperature decreases.
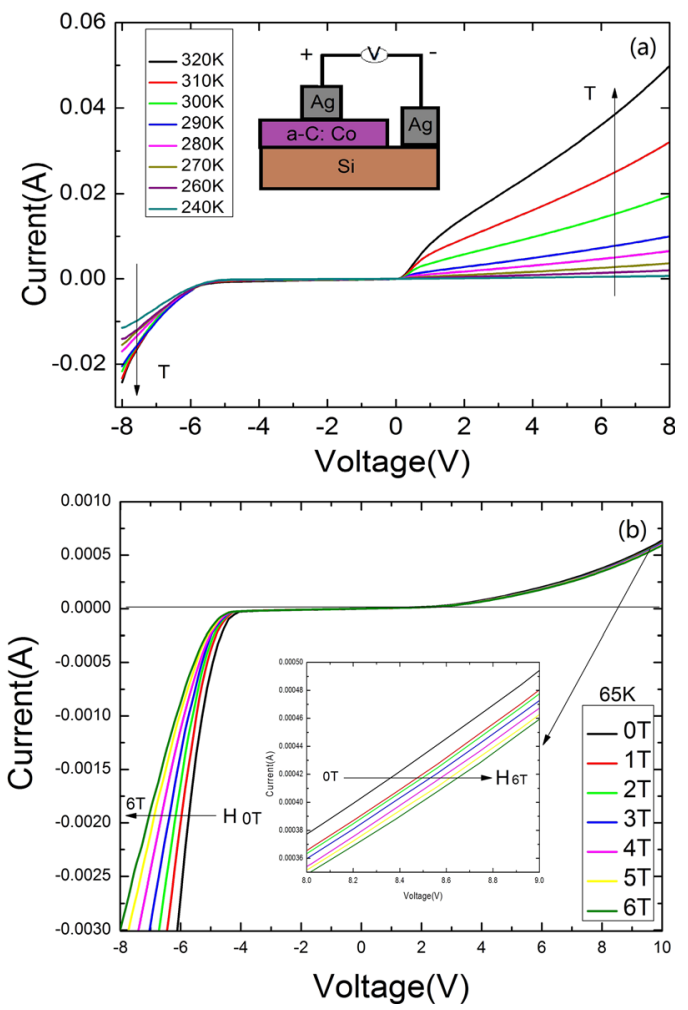

FIG. 3. (Color online) (a) $I-V$ characteristics of the a-C:Co/Si junction at different temperatures. The inset shows the schematic illustration of the electrical measurement. (b) The effect of magnetic fields on $I-V$ characteristics. The inset shows the amplification of a part of the $I-V$ curves at positive voltages. 

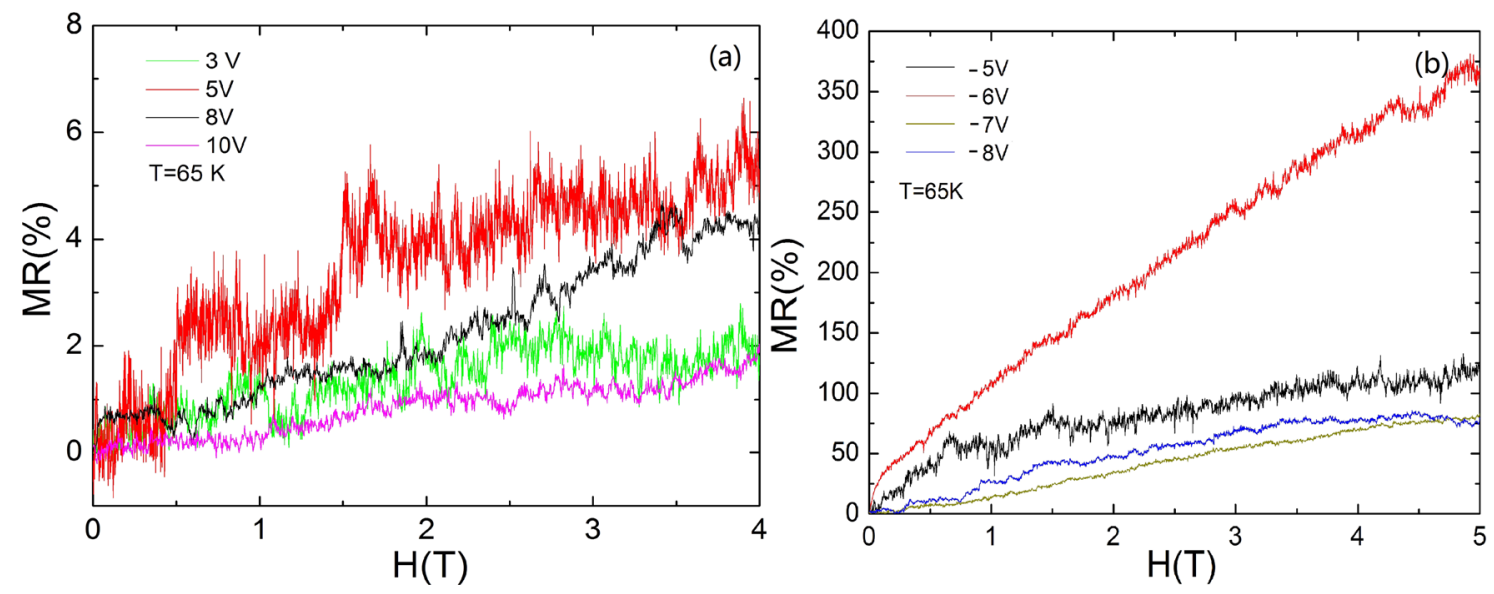

FIG. 4. (Color online) MR-H curves of the a-C:Co/Si junction dependent on (a) positive bias voltages, and (b) negative bias voltages.

Figure 3(b) shows $I-V$ characteristics of the a-C:Co/Si junction at $65 \mathrm{~K}$ when magnetic fields are placed vertically on the sample. The apparent PMR effect has been observed in both forward and reverse electric fields. The interesting result is that the magnetic field has a great effect on the $I-V$ characteristics under the reverse electric field, whereas the effect on the $I-V$ characteristics under the positive one is very small. The MR of the a-C:Co/Si junction is less than $6 \%$ at $H=5 \mathrm{~T}$ by measuring forward current values.

To provide a further insight into the PMR effect of the a-C:Co/Si junction, MR-H relations are exhibited in Figs. 4(a) and 4(b). It is observed that MR-H curves are apparently tuned by the bias voltages. Figure 4(a) shows that MR will reach a peak value at $V=5 \mathrm{~V}$ and $T=65 \mathrm{~K}$, which is consistent with the result of Tian et al. ${ }^{6}$ obtained at room temperature. Figure 4(b) shows that, under the reverse electric field, the a-C:Co/Si junction has a colossal PMR dependent on the bias voltage. The MR ( $-6 \mathrm{~V})$ reaches $100 \%$ at $H=1 \mathrm{~T}$ and over $350 \%$ at $H=5 \mathrm{~T}$. When the voltage value deviates from $-6 \mathrm{~V}$, the MR significantly decreases, but is still much higher than than in the positive voltage. This phenomenon indicates that there are two totally different PMR mechanisms dependent on the electric field direction. Although the $d$ electrons of Co atoms mainly have exchange interactions with $\mathrm{sp}^{2}$ hybridized electrons in the disordered carbon system, the PMR of the a-C:Co/Si junction is unlikely to be induced only by such exchange interactions, especially under the reverse electric field. ${ }^{6}$ A possible explanation is that the effect of the applied magnetic field on the Co ions leads to the transition from $\mathrm{sp}^{2}$ sites to $\mathrm{sp}^{3}$ sites, increases the barrier width, and then decreases carriers passing through the potential barrier by tunneling.

\section{CONCLUSIONS}

Co doped a-C films were deposited on the Si substrates by PLD at $400{ }^{\circ} \mathrm{C}$. Raman spectrum and $I-V$ characteristics have been investigated and analyzed. Our research mainly focuses on the PMR effect of the a-C:Co/Si junctions. The results show that the bias voltage has great effects on the MR-H relation. In addition, in the negative bias voltage, the a-C:Co/Si junction has much higher MR values than those in the positive bias voltage. A possible mechanism is provided to understand the anomalous PMR effect.

\section{ACKNOWLEDGMENTS}

The authors acknowledge the SEM results with the help of Mr. Wing Kin Ho. This work has been supported by a grant of the Research Grant Council of Hong Kong (Project No. HKU 702409P) and the CRCG Grant of the University of Hong Kong.

${ }^{1}$ F. Zhuge, W. Dai, C. L. He, A. Y. Wang, Y. W. Liu, M. Li, Y. H. Wu, P. Cui, and R.-W. Li, Appl. Phys. Lett. 96, 163505 (2010).

${ }^{2}$ M. Ma, Q. Xue, H. Chen, X. Zhou, D. Xia, C. Lv, and J. Xie, Appl. Phys. Lett. 97, 061902 (2010).

${ }^{3}$ A. M. M. Omer, S. Adhikari, S. Adhikary, H. Uchida, and M. Umeno, Appl. Phys. Lett. 87, 161912 (2005).

${ }^{4}$ X. Gao, Q. Xue, L. Hao, Q. Zheng, and Q. Li, Appl. Phys. Lett. 91, 122110 (2007).

${ }^{5}$ Q. Z. Xue and X. Zhang, Carbon 43, 760 (2005).

${ }^{6}$ P. Tian, X. Zhang, and Q. Z. Xue, Carbon 45, 1764 (2007).

${ }^{7}$ H. S. Hsu, P. Y. Chung, J. H. Zhang, S. J. Sun, H. Chou, H. C. Su, C. H. Lee, J. Chen, and J. C. A. Huang, Appl. Phys. Lett. 97, 032503 (2010).

${ }^{8}$ X. Zhang, X. Zhang, C. Wan, and L. Wu, Appl. Phys. Lett. 95, 022503 (2009).

${ }^{9}$ J. Tersoff, Phys. Rev. Lett. 61, 25 (1988).

${ }^{10}$ E. B. Halac, M. Reinoso, A. G. Dall'Asén, and E. Burgos, Phys. Rev. B 71, 115431 (2005).

${ }^{11}$ Y. Miyajima, A. A. D. T. Adikaari, S. J. Henley, J. M. Shannon, and S. R. P. Silva, Appl. Phys. Lett. 92, 152104 (2008).

${ }^{12}$ J. Gao, S. Y. Dai, and T. K. Li, Phys. Rev. B 67, 153403 (2003).

${ }^{13}$ F. X. Hu, J. Gao, and X. S. Wu, Phys. Rev. B 72, 064428 (2005).

${ }^{14}$ H. Qingrun and J. Gao, Appl. Phys. A 68, 343 (1999).

${ }^{15}$ J. Gao, W. H. Tamg, and T. C. Chui, Physica C 330, 33 (2000).

${ }^{16}$ M. A. Tamor and W. C. Vassell, J. Appl. Phys. 76, 6 (1994).

${ }^{17}$ X. Gao, Q. Xue, L. Hao, Q. Li, Q. Zheng, and K. Yan, Appl. Phys. A 371, 318 (2007). 